to consider how best to implement the Palliative Medicine aspect of the curriculum.

Method Our aim was to explore the extent of Palliative Medicine experience gained during Core Medical Training (CMT) from current specialty trainees in Wales to help guide implementation of the new curriculum. An online survey was sent to Welsh Palliative Medicine trainees, South Wales Palliative Medicine Specialty Doctors, and registrars on the General Medicine on call rotas in Abertawe Bro Morgannwg UHB in February 2019.

Results 17 trainees responded, 76\% felt they had received sufficient exposure to Palliative Medicine during CMT. Overall, responders felt confident in managing different aspects of palliative care, particularly recognising limited reversibility, the dying patient $(88 \%)$ and communication around end of life care issues (94\%). The best way to teach these palliative care skills is unresolved. Suggested effective methods included palliative care placement $(94 \%$ of responders agreed), small group teaching and communication skill workshops (59\%).

Discussion Our survey shows that the majority of responders were positive about their CMT experiences in Palliative Care. Some commented that most of these skills were learnt after starting specialty training, or from a CMT rotation in a hospice. Almost two-thirds of responders were Palliative Medicine middle grades suggesting more proactive approaches in seeking out these learning opportunities. Continued attention to placement opportunities, small group teaching and communication skills workshops would seem at present to be the most relevant response.

\section{QUALITY IMPROVEMENT PROJECT: IMPROVING JUNIOR DOCTORS' CONFIDENCE IN PRESCRIBING AT END OF LIFE FOR ACUTE INPATIENTS}

Laura Dewhirst, Hannah Hudson. Shrewsbury and Telford Hospital Trusts

10.1136/spcare-2020-PCC.37

Background Junior doctors are often at the forefront of prescribing for acute inpatients. It is imperative that they are equipped with the knowledge and confidence to prescribe appropriately for patients at the end of their life. We felt that there was reduced awareness of the need for individualised prescribing for patients with relatively common chronic diseases such as Parkinson's disease and epilepsy. Recognising that prescribing end of life medications for patients with such diseases can differ, we looked to improve symptom management by focussing on improving end of life prescribing.

Methods A pre-intervention questionnaire was used to assess subjective confidence levels in prescribing anticipatory medications, syringe drivers, and individualised prescribing for patients with Parkinson's disease, end-stage renal disease, epilepsy, end-stage heart failure, and diabetes mellitus. Based on these findings a prescribing handbook was produced, incorporating consultant advice from relevant specialities. Teaching sessions were carried out using case-based discussions alongside the handbook content. A local press release facilitated dissemination of the handbook. A post-intervention questionnaire was then distributed to reassess prescribers' confidence levels.
Results Twenty-five allied health professionals (mainly foundation doctors) completed the questionnaires. The preintervention questionnaire found that the majority were confident in prescribing anticipatory medication but were significantly less confident in prescribing syringe drivers and in individualised prescribing. Following formal education and release of the prescribing handbook, higher confidence levels were reported throughout all themes of the questionnaire.

Conclusions This quality improvement project has significantly increased prescribers' confidence levels in end of life prescribing. It has also increased awareness of the need for alternative approaches to prescribing for certain individuals and diseases at the end of life. It was stressed that the handbook is to be used as a guide and should not replace the need to escalate concerns to the relevant specialities and the in-house palliative care team.

\section{SIMPALL AN IN-SITU SIMULATION COURSE ON PALLIATIVE CARE FOR THE EMERGENCY DEPARTMENT}

S Edwards, L Keillor, S Krauze, R Singh, K Murray, J Grant. University Hospitals of Leicester

\subsection{6/spcare-2020-PCC.38}

Aims/Objectives/Background Emergency Medicine is a unique speciality often meeting people at the worse moments of their life. Death is an everyday occurrence, and with that comes the skills needed to talk to patients and families about when their end of life may be nearing. The Royal College of Emergency Medicine's guideline suggests doctors need to have the skills to talk to these patients. We developed a full day course called SimPall which is incorporates some lecture-based teaching, and then in-situ simulation within the emergency department. Our aim was to gather feedback to see what educational benefit this brought to our EM staff.

Methods/Design Following some teaching around difficult conversations, do not attempt cardio pulmonary resuscitation and managing palliative emergencies we then gave the participants all 3 of our in-situ simulations, in two groups. The participants were not aware of what simulations they got. The three simulations based on real ED patients were; Scenario 1 was the end stage COPD patient who is not for any further interventions. Scenario 2 is of a very frail patient, with pneumonia who has multiple comorbidities. Scenario 3 was a massive upper gastrointestinal bleed with known oesophageal cancer. All participants were debriefed, and feedback recorded. All participants were offered the chance to discuss with a senior if they had any worries about the scenarios.

Results/Conclusions This teaching day was in April 2019. 13 people including doctors and advanced nurse practitioners attended the day. All were involved in each of the scenarios and their feedback was collated. Overall the feedback for all the simulations was positive. $76 \%$ of participants felt their knowledge had improved from lacking confidence to being fairly or very confident after the simulations. Written comments suggest that participants feel they would benefit from more teaching and exposure of this. We are looking at repeating this day. 